\title{
Sequencing games with repeated players
}

\author{
Arantza Estévez-Fernández • Peter Borm • \\ Pedro Calleja • Herbert Hamers
}

Published online: 18 September 2007

(C) Springer Science+Business Media, LLC 2007

\begin{abstract}
Two classes of one machine sequencing situations are considered in which each job corresponds to exactly one player but a player may have more than one job to be processed, so called RP(repeated player) sequencing situations. In max-RP sequencing situations it is assumed that each player's cost function is linear with respect to the maximum completion time of his jobs, whereas in min-RP sequencing situations the cost functions are linear with respect to the minimum completion times. For both classes, following explicit procedures to go from the initial processing order to an optimal order for the coalition of all players, equal gain splitting rules are defined. It is shown that these rules lead to core elements of the associated RP sequencing games. Moreover, it is seen that min-RP sequencing games are convex.
\end{abstract}

Keywords Cooperative game theory $\cdot$ Sequencing $\cdot$ Equal gain splitting $\cdot$ Core $\cdot$ Convexity

\section{Introduction}

Scheduling problems were first studied from an interactive cooperative point of view by Curiel et al. (1989) in the framework of one-machine sequencing with an initial processing

We thank two referees for their valuable suggestions for improvement.

Financial support for P. Calleja has been given by the Ministerio de Educación y Ciencia and FEDER under grant SEJ2005-02443/ECON, and by the Generalitat de Catalunya through a BE grant from AGAUR and grant 2005SGR00984.

A. Estévez-Fernández $(\bowtie)$

Department of Econometrics and OR, Vrije Universiteit, De Boelelaan 1105, 1081 HV Amsterdam, The Netherlands

e-mail: A.E.Fernandez@uvt.nl

P. Borm · H. Hamers

P.O. Box 90153, 5000 LE Tilburg, The Netherlands

P. Calleja

Department of Economic, Financial, and Actuarial Mathematics, University of Barcelona,

Av. Diagonal 690, 08034 Barcelona, Spain 
order on the jobs. Identifying jobs with players and introducing cost functions for the players dependent on the completion time of their jobs, an associated cooperative game is defined in which the value of a coalition reflects the maximum cost savings this coalition can achieve by reordering their jobs from their initial position to a feasible, optimal one. Different types of sequencing games have been studied in the literature depending on the structure of the underlying cost functions, the number of machines, further restrictions such as ready times and due dates, etc. For a survey we refer to Curiel et al. (2002). The common feature in this stream of literature is the assumption that each job is of interest to exactly one player and that each player has exactly one job to be processed. An exception is the recent contribution by Calleja et al. (2006). Here, the latter assumption is dropped, so a job may correspond to several players and a player may have interest in more than one job. It is shown that the corresponding sequencing games are balanced if the underlying cost functions satisfy a specific type of additivity with respect to the initial order.

In the current paper, we focus on the specific subclass of RP (repeated players) sequencing situations in which (just as in the classical approach) each job corresponds to exactly one player, but a player may have more than one job to be processed. Moreover, we restrict attention to two types of RP sequencing situations and their related games. In max-RP sequencing situations it is assumed that each player's cost function is linear with respect to the maximum completion time of his jobs, whereas in min-RP sequencing situations the cost function of a player is linear with respect to the minimum completion time of his jobs. It was already pointed out in Calleja et al. (2006) that both types of cost functions satisfy the additivity condition, so the corresponding games are balanced. Next, we offer two motivating examples for these types of cost functions.

In a garage, a car may need more than one reparation (change of tires, of oil, etc). Here, it seems reasonable to assume that each repair job not only has a certain fixed cost but the owner also incurs variable costs that are proportional to the total time that the car has to spend in the garage, i.e., to the completion time of the last reparation carried out in the car. Note that the reparations in the car are complementary since the car cannot leave the garage until it is completely repaired. Moreover, the different jobs of a player need not be carried out consecutively due to specific other priority considerations (e.g. first come first served) the garage may have on the specific individual tasks.

The classrooms of a faculty are equipped with an overhead projector and a beamer. If one of the devices breaks down, lecturers have to report to the maintenance service for the device(s) to be repaired, incurring a fixed cost for each reparation. A lecturer needs at least one of the devices to start the lesson. Hence, when both devices are out of order, there is an extra variable cost which is proportional to the time that she has to wait until she can start her lecture, i.e., until one of the devices is fixed. Note that the reparations in this setting are substitutes since the lecturer can start her lecture as soon as one of the reparations is carried out.

The contributions of the current paper are the following. For both max-RP and min-RP sequencing situations, explicit procedures are devised to go from the initial order on all jobs to an optimal one. Following the steps of this procedure an EGS (equal gain splitting) mechanism is adopted to construct an allocation rule for the maximal cost savings of the grand coalition. It is shown that this EGS-allocation is in the core of the associated game, and that it in fact is PMAS-extendable (cf. Sprumont 1990). In particular, this implies that for calculating a core allocation one does not need the data on all coalitional values. In addition, it is shown that min-RP sequencing games are convex.

The structure of the paper is as follows. Section 2 recalls some basic game theoretic notions and provides the formal definition of RP sequencing situations and related games. 
Section 3 considers max-RP sequencing whereas Sect. 4 analyzes min-RP sequencing. An appendix contains the lemmas used in the proofs of the main results.

\section{Preliminaries}

A cooperative TU-game in characteristic function form is an ordered pair $(N, v)$, where $N$ is a finite set (the set of players) and $v: 2^{N} \rightarrow \mathbb{R}$ satisfies $v(\emptyset)=0$. The core of a cooperative TU-game $(N, v)$ is defined by

$$
\operatorname{Core}(v)=\left\{x \in \mathbb{R}^{N} \mid \sum_{i \in N} x_{i}=v(N), \sum_{i \in S} x_{i} \geq v(S) \text { for all } S \in 2^{N}\right\},
$$

i.e., the core is the set of efficient allocations of $v(N)$ such that there is no coalition with an incentive to split off. A game is said to be balanced (see Bondareva 1963 and Shapley 1967) if the core is nonempty.

An important subclass of balanced games is the class of convex games (Shapley 1971). A game $(N, v)$ is said to be convex if

$$
v(T \cup\{i\})-v(T) \geq v(S \cup\{i\})-v(S)
$$

for every $i \in N$ and for every $S \subset T \subset N \backslash\{i\}$. It is known that convex games are totally balanced and that the core is the convex hull of all marginal vectors.

A sequencing situation is a 4-tuple $\left(N, \sigma_{0}, p, c\right)$, where $N=\{1, \ldots, n\}$ is the set of players (or jobs), $\sigma_{0}: N \rightarrow\{1, \ldots, n\}$ is a bijection that represents the initial order on the jobs (job $i$ is in position $\left.\sigma_{0}(i)\right), p \in \mathbb{R}^{N}$ is the vector of processing times of the jobs, and $c=\left(c_{i}\right)_{i \in N}$ is the vector of cost functions of the players depending on the completion time of their jobs, so $c_{i}:[0,+\infty) \rightarrow \mathbb{R}$. Costs are assumed to be linear, i.e., $c_{i}(t)=\alpha_{i} t$ with $\alpha_{i}>0$. Alternatively, a sequencing situation $\left(N, \sigma_{0}, p, c\right)$ is denoted by $\left(N, \sigma_{0}, p, \alpha\right)$ with $\alpha \in \mathbb{R}_{++}^{N}$. Let $\Pi(N)$ denote the set of all possible orders of the jobs. For $\sigma \in \Pi(N), \sigma(k)$ indicates the position in which job $k$ is processed. Sometimes we denote $\sigma=(\sigma(1) \ldots \sigma(n))$. Given an order $\sigma \in \Pi(N)$ the jobs are processed in a semi-active way, i.e., there is no job that could be processed earlier without altering the processing order. Therefore, the completion time of player $i$ is given by $C_{i}^{\sigma}=\sum_{j \in N: \sigma(j) \leq \sigma(i)} p_{j}$. For simplicity we denote $c_{i}\left(C_{i}^{\sigma}\right)$ by $c_{i}(\sigma)$.

Given a sequencing situation the associated sequencing game, $\left(N, v_{C}\right)$, is defined by

$$
v_{C}(S)=\max _{\sigma \in \mathcal{F}(S)}\left(c_{S}\left(\sigma_{0}\right)-c_{S}(\sigma)\right)
$$

for every $S \subset N$, where, for all $\sigma \in \Pi(N), c_{S}(\sigma)=\sum_{i \in S} c_{i}(\sigma)$ and $\mathcal{F}(S)$ is the set of feasible orders for coalition $S$. An order $\sigma \in \Pi(N)$ is said to be feasible for $S$ if $P_{j}(\sigma)=$ $P_{j}\left(\sigma_{0}\right)$ for all $j \in N \backslash S$, where $P_{j}(\sigma)=\{k \in N \mid \sigma(k)<\sigma(j)\}$ is the set of predecessors of $j$ with respect to $\sigma$. Note that feasible orders only allow reordering within connected components of $S$ with respect to $\sigma_{0}$. The set of connected components of $S$ with respect to $\sigma_{0}$ is denoted $S / \sigma_{0}$. Assuming $\sigma_{0}=\left(\begin{array}{llll}1 & 2 \ldots n\end{array}\right)$ the associated coalitional values can be expressed as

$$
v_{C}(S)=\sum_{T \in S / \sigma_{0}} \sum_{i, j \in T: i<j} g_{i j}
$$


for every $S \subset N$, where $g_{i j}=\max \left\{0, \alpha_{j} p_{i}-\alpha_{i} p_{j}\right\}$ is the cost savings that players $i$ and $j$ can achieve by means of a neighbor switch when $i$ is in front of $j$ (cf. Curiel et al. 1989).

Since an optimal order for the grand coalition can be derived from the initial order by non-negative neighbor switches, a natural allocation rule in sequencing situations is provided by the equal gain splitting rule or EGS rule introduced in Curiel et al. (1989), where the cost savings attained by neighbor switches are divided equally among the players involved. Formally,

$$
\operatorname{EGS}_{i}\left(N, \sigma_{0}, p, \alpha\right)=\frac{1}{2} \sum_{j=1}^{i-1} g_{j i}+\frac{1}{2} \sum_{j=i+1}^{n} g_{i j}
$$

for every $i \in N$.

Sequencing games are convex and the EGS rule provides a core allocation.

An $R P$ (repeated players) sequencing situation is a 6-tuple $\left(N, M, J, \sigma_{0}, p, c\right)$, where $N=\{1, \ldots, n\}$ is the set of players, $M$ is the finite set of jobs, $J: N \rightarrow M$ is a correspondence where $J(i)$ denotes the nonempty set of jobs in which player $i$ is involved with the extra condition that $\bigcup_{i \in N} J(i)=M$ and $J(i) \cap J(j)=\emptyset$ for all $i, j \in N, i \neq j$, $\sigma_{0}: M \rightarrow\{1, \ldots,|M|\}$ is a bijection representing the initial order on the jobs, $p \in \mathbb{R}^{M}$ is the vector of processing times of the jobs, and $c=\left(c_{i}\right)_{i \in N}$ is the vector of cost functions associated to the players. Let $\Pi(M)$ denote the set of all bijections $\sigma: M \rightarrow\{1, \ldots,|M|\}$. Given an order $\sigma \in \Pi(M)$ it is assumed that the jobs are processed in a semi-active way.

Given an RP sequencing situation $\left(N, M, J, \sigma_{0}, p, c\right)$ the associated $R P$ sequencing game $(N, v)$ is defined by

$$
v(S)=\max _{\sigma \in \mathcal{A}(S)}\left(c_{S}\left(\sigma_{0}\right)-c_{S}(\sigma)\right)
$$

for every $S \subset N$, where for all $\sigma \in \Pi(M), c_{S}(\sigma)=\sum_{i \in S} c_{i}(\sigma)$ and $\mathcal{A}(S)$ is the set of admissible orders for coalition $S$. An order $\sigma \in \Pi(M)$ is said to be admissible for $S$ if $P_{d}(\sigma)=P_{d}\left(\sigma_{0}\right)$ for all $d \notin \bigcup_{i \in S} J(i)$, where $P_{d}(\sigma)=\{e \in M \mid \sigma(e)<\sigma(d)\}$ is the set of predecessors of job $d$ with respect to $\sigma$. Note that if an order is admissible for $S$, the completion time of each job belonging to a player in $N \backslash S$ does not change. Moreover, only within connected components of $\bigcup_{i \in S} J(i)$ w.r.t. $\sigma_{0}$, jobs can be reordered.

It has been shown in Calleja et al. (2006) that RP sequencing games are balanced if the cost functions of the players are "additive with respect to the initial order". Formally, a cost function $c_{i}$ is additive with respect to $\sigma_{0}$ if for all $L_{1}, L_{2} \subset M$ with $L_{1} \cap L_{2}=\emptyset$, all $\rho \in \mathcal{F}\left(L_{1}\right)$ and $\tau \in \mathcal{F}\left(L_{2}\right)$, it follows that

$$
c_{i}\left(\sigma_{0}\right)-c_{i}(\pi)=\left(c_{i}\left(\sigma_{0}\right)-c_{i}(\rho)\right)+\left(c_{i}\left(\sigma_{0}\right)-c_{i}(\tau)\right),
$$

where $\pi \in \mathcal{F}\left(L_{1} \cup L_{2}\right)$ is such that

$$
\pi(d)= \begin{cases}\rho(d) & \text { if } d \in L_{1} \\ \tau(d) & \text { if } d \in L_{2}, \\ \sigma_{0}(d) & \text { if } d \in M \backslash\left(L_{1} \cup L_{2}\right) .\end{cases}
$$

Here, $\mathcal{F}(K)$ denotes the set of feasible orders for $K \subset M$ and an order $\sigma \in \Pi(M)$ of jobs is called feasible for $K \subset M$ if $P_{d}(\sigma)=P_{d}\left(\sigma_{0}\right)$ for all $d \in M \backslash K$. 


\section{Max-RP sequencing}

In this section we consider max-RP sequencing situations and associated games.

A max-RP sequencing situation is an RP sequencing situation where $c_{i}(\sigma)=$ $\alpha_{i} \max _{d \in J(i)}\left\{C_{d}^{\sigma}\right\}$ for some $\alpha_{i}>0$, for every $i \in N$ and all $\sigma \in \Pi(M)$. Usually, a max$\mathrm{RP}$ sequencing situation like this is described by $\left(N, M, J, \sigma_{0}, p, \alpha\right)$ with $\alpha \in \mathbb{R}_{++}^{N}$. It has been pointed out in Calleja et al. (2006) that the above type of cost functions satisfies the additivity condition needed for balancedness of the associated sequencing game.

Given an order $\sigma \in \Pi(M)$, we denote by $l_{i}^{\sigma} \in M$ the job of $i \in N$ that is processed in last position according to $\sigma$, i.e., $\sigma\left(l_{i}^{\sigma}\right) \geq \sigma(d)$ for all $d \in J(i)$. Note that an order $\sigma \in \Pi(M)$ induces an order $\bar{\sigma} \in \Pi(N)$ on the players in the following way: $\bar{\sigma}(i)<\bar{\sigma}(j)$ if and only if $\sigma\left(l_{i}^{\sigma}\right)<\sigma\left(l_{j}^{\sigma}\right)$. Throughout this section we assume w.l.o.g. that $\sigma_{0} \in \Pi(M)$ is such that $\bar{\sigma}_{0}=\left(\begin{array}{llll}1 & 2 & \ldots & n\end{array}\right)$ and we write $l_{i}=l_{i}^{\sigma_{0}}$. Hence, $i<j$ if and only if $\sigma_{0}\left(l_{i}\right)<\sigma_{0}\left(l_{j}\right)$.

We say that the jobs of player $i$ are clustered according to an order $\sigma \in \Pi(M)$ if they are processed consecutively, i.e., if $d_{1}, d_{2} \in J(i)$ and $\sigma\left(d_{1}\right)<\sigma(e)<\sigma\left(d_{2}\right)$ imply that $e \in J(i)$. It is easy to see that all jobs of a player are clustered in an optimal order for max-RP sequencing situations. To derive an optimal order on all jobs, we next turn to classical sequencing: the optimal order of the clusters is in non-decreasing order of urgencies (cf. Smith 1956). Here, the urgency of a cluster obviously is the quotient of cost coefficient $\alpha_{i}$ of the corresponding player and the processing time of the cluster which is given by $\sum_{d \in J(i)} p_{d}$.

An explicit procedure to derive an optimal order for $M$ from the initial order $\sigma_{0}$ by nonnegative switches is described in the following way.

First, we put all the jobs of player $n$ at the back of the queue ${ }^{1}$. After this, all jobs of player $n-1$ are clustered in front of the jobs of player $n$, and so on. Note that the cost savings induced on $i$ by clustering the jobs of $j(i<j)$ during this step are given by: $b_{i j}^{N}=$ $\alpha_{i} \sum_{e \in J(j): \sigma_{0}(e)<\sigma_{0}\left(l_{i}\right)} p_{e}$.

Second, consider the (remaining) classical sequencing situation (on the constructed clusters) given by $\left(N, \bar{\sigma}_{0}, q, \alpha\right)$ with $q \in \mathbb{R}^{N}$ such that $q_{i}=\sum_{d \in J(i)} p_{d}$. The cost savings in this step can be obtained by non-negative (cluster) neighbor switches and equal $\sum_{i, j \in N: i<j} g_{i j}^{N}$ with $g_{i j}^{N}=\max \left\{0, \alpha_{j} q_{i}-\alpha_{i} q_{j}\right\}$.

Summarizing, the total maximal cost savings obtainable by means of cooperation are

$$
v(N)=\sum_{i=1}^{n-1} \sum_{j=i+1}^{n} r(i, j, N)
$$

where

$$
r(i, j, N):=b_{i j}^{N}+g_{i j}^{N}
$$

for all $i, j \in N$ with $i<j$.

Adopting the equal gain splitting mechanism in the procedure above we can define the $\max -E G S$ rule in the following way:

$$
\max -E_{i}\left(N, M, J, \sigma_{0}, p, \alpha\right)=\frac{1}{2} \sum_{j=i+1}^{n} r(i, j, N)+\frac{1}{2} \sum_{j=1}^{i-1} r(j, i, N) .
$$

\footnotetext{
${ }^{1}$ Remember that we assume $\sigma_{0}\left(l_{n}\right)>\sigma_{0}\left(l_{i}\right)$ for all $i \in N, i \neq n$.
} 
Next, we show that the max-EGS rule leads to core elements of the associated max-RP sequencing game. With this purpose, we first give an explicit construction and expression for coalitional values in a max-RP sequencing game similar to the derivation of $v(N)$ provided above. For this, we need some extra notation. Given $S \subset N$ let $\left[J(S) / \sigma_{0}\right]=\left\{U_{1}, \ldots, U_{u}\right\}$ be the set of maximal connected components of the set of jobs $J(S)$ such that $U_{r} \cap\left\{l_{i}\right\}_{i \in S} \neq$ $\emptyset$ for all $r$. The collection $\left\{U_{1}, \ldots, U_{u}\right\}$ is called the induced job collection of $S$ by $\sigma_{0}$. Associated to each $U_{r}$ we define $S_{r}$ by $S_{r}:=\left\{i \in S \mid l_{i} \in U_{r}\right\}$. Observe that $\left\{S_{1}, \ldots, S_{u}\right\}$ is a partition of $S$. We call this partition the induced partition of $S$ by $\sigma_{0}$.

Now, consider $i \in S_{r}$ and $j \in S$ with $i<j$. We denote by $b_{i j}^{S_{r}}$ the cost savings induced on $i$ when moving the jobs of player $j$ that are in $U_{r}$ to be clustered to the back, i.e., $b_{i j}^{S_{r}}=\alpha_{i} \sum_{\substack{e \in J(j) \cap U_{r} \\ \sigma_{0}(e)<\sigma_{0}\left(l_{i}\right)}} p_{e}$.

Moreover, with $i, j \in S_{r}$ and $i<j$ we denote by $g_{i j}^{S_{r}}$ the cost savings obtainable by means of a neighbor switch between the clusters corresponding to $i$ and $j$ within $U_{r}$, i.e., $g_{i j}^{S_{r}}=\max \left\{0, \alpha_{j} \sum_{d \in J(i) \cap U_{r}} p_{d}-\alpha_{i} \sum_{e \in J(j) \cap U_{r}} p_{e}\right\}$.

For $i, j \in S$ with $i<j$ we define

$$
r(i, j, S)= \begin{cases}b_{i j}^{S_{r}}+g_{i j}^{S_{r}}, & \text { if } i, j \in S_{r} ; \\ b_{i j}^{S_{r}}, & \text { if } i \in S_{r}, j \notin S_{r} .\end{cases}
$$

Note that $b_{i j}^{S_{r}}$ and $g_{i j}^{S_{r}}$ are nonnegative and therefore $r(i, j, S)$ is nonnegative.

It is readily established that the coalitional value $v(S)$ in the corresponding max-RP sequencing game is given by

$$
v(S)=\sum_{i \in S} \sum_{j \in S: i<j} r(i, j, S) .
$$

Theorem 3.1 For any max-RP sequencing game, the max-EGS rule provides a core element.

Proof Let $\left(N, M, J, \sigma_{0}, p, \alpha\right)$ be a max-RP sequencing situation and let $(N, v)$ be the associated max-RP sequencing game.

Efficiency follows by definition. Next, we show that the rule is stable. Let $S \subset N$, then

$$
\begin{aligned}
& \sum_{i \in S} \max -\mathrm{EGS}_{i}\left(N, M, J, \sigma_{0}, p, \alpha\right) \\
& \quad=\sum_{i \in S}\left[\frac{1}{2} \sum_{j=i+1}^{n} r(i, j, N)+\frac{1}{2} \sum_{j=1}^{i-1} r(j, i, N)\right] \\
& \quad=\sum_{i \in S} \sum_{j \in S: i<j} r(i, j, N)+\frac{1}{2} \sum_{i \in S} \sum_{j \in N \backslash S: i<j} r(i, j, N)+\frac{1}{2} \sum_{i \in S} \sum_{j \in N \backslash S: j<i} r(j, i, N) \\
& \quad \geq \sum_{i \in S} \sum_{j \in S: i<j} r(i, j, N) \geq \sum_{i \in S} \sum_{j \in S: i<j} r(i, j, S)=v(S),
\end{aligned}
$$

where the last inequality follows by Lemma 5.1 .

Note that in fact the max-EGS core allocation is PMAS extendable (cf. Sprumont 1990) by considering the max-EGS allocations for all subgames, and the use of Lemma 5.1.

In general, max-RP sequencing games need not be convex (cf. Example 3.7 in Calleja et al. 2006). 


\section{Min-RP sequencing}

In this section we analyze min-RP sequencing situations and related games.

A min-RP sequencing situation is an RP sequencing situation where $c_{i}(\sigma)=$ $\alpha_{i} \min _{d \in J(i)}\left\{C_{d}^{\sigma}\right\}$ for some $\alpha_{i}>0$, for every $i \in N$ and all $\sigma \in \Pi(M)$. Usually, a min-RP sequencing situation like this is described by $\left(N, M, J, \sigma_{0}, p, \alpha\right)$ with $\alpha \in \mathbb{R}_{++}^{N}$. It has been pointed out in Calleja et al. (2006) that also this type of cost functions satisfies the additivity condition needed for balancedness of the associated sequencing game.

Given an order $\sigma \in \Pi(M)$, we denote by $f_{i}^{\sigma} \in M$ the job of $i \in N$ that is processed in first position according to $\sigma$, i.e., $\sigma\left(f_{i}^{\sigma}\right) \leq \sigma(d)$ for all $d \in J(i)$. Note that an order $\sigma$ on the jobs induces an order $\tilde{\sigma} \in \Pi(N)$ on the players in the following way: $\tilde{\sigma}(i)<\tilde{\sigma}(j)$ if and only if $\sigma\left(f_{i}^{\sigma}\right)<\sigma\left(f_{j}^{\sigma}\right)$. Throughout this section we assume w.l.o.g. that $\sigma_{0}$ is such that $\tilde{\sigma}_{0}=\left(\begin{array}{lll}1 & 2 & \ldots n\end{array}\right)$ and we write $f_{i}=f_{i}^{\sigma_{0}}$. Hence, $i<j$ if and only if $\sigma_{0}\left(f_{i}\right)<\sigma_{0}\left(f_{j}\right)$.

It is easy to see that for min-RP sequencing situations in every optimal order on $M$ the first $n$ jobs belong to different players. Moreover, the first job of a player is one of its jobs with minimum processing time. Finally, the first $n$ jobs are processed in decreasing order with respect to their urgencies.

An optimal order can be constructed from $\sigma_{0}$ by non-negative switches in the following way. First, we put all the jobs of player 1 that are not $f_{1}$ and are in front of $f_{n}$ at the back of the queue ${ }^{2}$. Second, we do the same with all the jobs of player 2 that are not $f_{2}$ and are in front of $f_{n}$, and so on. Once we finish this step, the first $n$ jobs of the queue belong to different players. Observe that the cost savings induced on $j$ by moving jobs of player $i$ $(i<j)$ to the back are given by: $\gamma_{i j}^{N}=\alpha_{j} \sum_{d \in J(i) \backslash\left\{f_{i}\right\}: \sigma_{0}(d)<\sigma_{0}\left(f_{j}\right)} p_{d}$.

Next, we switch $f_{i}{ }^{3}$ with the job of player $i$ that has shortest processing time, if necessary, in the order $1,2, \ldots, n$. The cost savings induced on $j$ by the internal job switch of $i$ (with $i \leq j)$ in this step are: $\delta_{i j}^{N}=\alpha_{j}\left(p_{f_{i}}-\min _{d \in J(i)}\left\{p_{d}\right\}\right)$.

Now, consider the (remaining) classical sequencing situation (on these first $n$ jobs) given by $\left(N, \tilde{\sigma}_{0}, q, \alpha\right)$ with $q \in \mathbb{R}^{N}$ such that $q_{i}=\min _{d \in J(i)} p_{d}$. Clearly, the maximal cost savings in this third step equal $\sum_{i, j \in N: i<j} g_{i j}^{N}$ with $g_{i j}^{N}=\max \left\{0, \alpha_{j} q_{i}-\alpha_{i} q_{j}\right\}$. Summarizing, the total maximal cost savings obtainable by means of cooperation are

$$
v(N)=\sum_{i=1}^{n-1} \sum_{j=i+1}^{n} s(i, j, N)+\sum_{i=1}^{n} s(i, N),
$$

where

$$
s(i, N)=\delta_{i i}^{N}
$$

and

$$
s(i, j, N)=\gamma_{i j}^{N}+\delta_{i j}^{N}+g_{i j}^{N}
$$

for all $i, j \in N$ with $i<j$.

\footnotetext{
${ }^{2}$ Remember that we have assumed that $\sigma_{0}\left(f_{1}\right)<\sigma_{0}\left(f_{j}\right)$ for all $j \in N, j \neq 1$.

${ }^{3}$ Note that for the order $\sigma \in \Pi(M)$ derived of the first step we have that $f_{i}=f_{i}^{\sigma}$ for all $i \in N$.
} 
Adopting the equal gain splitting mechanism in the procedure above, we can define the $\min -E G S$ rule in the following way

$$
\min -\mathrm{EGS}_{i}\left(N, M, J, \sigma_{0}, p, \alpha\right)=\frac{1}{2} \sum_{j=1}^{i-1} s(j, i, N)+\frac{1}{2} \sum_{j=i+1}^{n} s(i, j, N)+s(i, N) .
$$

The min-EGS rule leads to core allocations of the associated min-RP sequencing games. To show this, we first give an explicit construction and expression for coalitional values in a min-RP sequencing game similarly to max-RP games. For this, we first introduce some notation. Given $S \subset N$ let $\left[J(S) / \sigma_{0}\right]=\left\{U_{1}, \ldots, U_{u}\right\}$ be the set of maximal connected components of $J(S)$ such that $U_{r} \cap\left\{f_{i}\right\}_{i \in S} \neq \emptyset$ for all $r$. The collection $\left\{U_{1}, \ldots, U_{u}\right\}$ is called the induced job collection of $S$ by $\sigma_{0}$. Associated to each $U_{r}$ we define $S_{r}$ by $S_{r}:=\left\{i \in S \mid f_{i} \in U_{r}\right\}$. Observe that $\left\{S_{1}, \ldots, S_{u}\right\}$ is a partition of $S$. We call this partition the induced partition of $S$ by $\sigma_{0}$. Note that $\left\{U_{1}, \ldots, U_{u}\right\}$ and $\left\{S_{1}, \ldots, S_{u}\right\}$ have a similar interpretation than in max-RP sequencing situations by replacing $l_{i}$ by $f_{i}$. However, we use the same notation since there is no reason for misinterpretation.

Now, consider $i \in S_{r}, j \in S_{t}$ with $i<j$. We denote by $\gamma_{i j}^{S_{t}}$ the cost savings that player $j$ obtains when the jobs of player $i$ within $U_{t}$ (unequal to $f_{i}$ ) that are in front of $f_{j}$ are moved to the back of $U_{t}$, i.e., $\gamma_{i j}^{S_{t}}=\alpha_{j} \sum_{\substack{d \in\left(J(i) \backslash\left\{f_{i}\right) \cap U_{t} \\ \sigma_{0}(d)<\sigma_{0}\left(f_{j}\right)\right.}} p_{d}$.

Next, consider $i, j \in S_{r}$ with $i \leq j$. With $\delta_{i j}^{S_{r}}$ we symbolize the cost savings that player $j$ obtains when player $i$ switches its first job with the one with minimum processing time in $U_{r}$, i.e., $\delta_{i j}^{S_{r}}=\alpha_{j}\left(p_{f_{i}}-\min _{d \in J(i) \cap U_{r}}\left\{p_{d}\right\}\right)$.

Finally, take $i, j \in S_{r}$ with $i<j$. By $g_{i j}^{S_{r}}$ we denote the cost savings obtainable by means of a neighbor switch between the two jobs of players $i$ and $j$ within $U_{r}$ with shortest processing times, i.e., $g_{i j}^{S_{r}}=\max \left\{0, \alpha_{j} \min _{d \in J(i) \cap U_{r}}\left\{p_{d}\right\}-\alpha_{i} \min _{e \in J(j) \cap U_{r}}\left\{p_{e}\right\}\right\}$.

Let $S \subset N$. For $i, j \in S$ and $i<j$ define

$$
s(i, j, S)= \begin{cases}\gamma_{i j}^{S_{r}}+\delta_{i j}^{S_{r}}+g_{i j}^{S_{r}}, & \text { if } i, j \in S_{r} \\ \gamma_{i j}^{S_{t}}, & \text { if } i \in S_{r}, j \in S_{t}, \text { and } t \neq r\end{cases}
$$

and for $i \in S_{r}$,

$$
s(i, S)=\delta_{i i}^{S_{r}} .
$$

Note that $\gamma_{i j}^{S_{r}}, \delta_{i j}^{S_{r}}, g_{i j}^{S_{r}}$, and $\delta_{i i}^{S_{r}}$ are nonnegative and therefore $s(i, j, S)$ and $s(i, S)$ are also nonnegative.

Then, it readily follows that the coalitional value $v(S)$ in the corresponding min-RP sequencing game is given by

$$
v(S)=\sum_{i \in S} \sum_{j \in S: i<j} s(i, j, S)+\sum_{i \in S} s(i, S)
$$

Theorem 4.1 For any min-RP sequencing game, the min-EGS rule provides a core element.

Proof Let $\left(N, M, J, \sigma_{0}, p, \alpha\right)$ be a min-RP sequencing situation and let $(N, v)$ be the associated min-RP sequencing game. 
Efficiency follows by definition. Next, it is shown that the rule is stable. Let $S \subset N$, then

$$
\begin{aligned}
\sum_{i \in S} & \min -\mathrm{EGS}_{i}\left(N, M, J, \sigma_{0}, p, \alpha\right) \\
= & \sum_{i \in S}\left[\frac{1}{2} \sum_{j=1}^{i-1} s(j, i, N)+\frac{1}{2} \sum_{j=i+1}^{n} s(i, j, N)+s(i, N)\right] \\
= & \sum_{i \in S} \sum_{j \in S: j<i} s(j, i, N)+\frac{1}{2} \sum_{i \in S} \sum_{j \in N \backslash S: j<i} s(j, i, N) \\
& +\frac{1}{2} \sum_{i \in S} \sum_{j \in N \backslash S: i<j} s(i, j, N)+\sum_{i \in S} s(i, N) \\
\geq & \sum_{i \in S} \sum_{j \in S: j<i} s(j, i, N)+\sum_{i \in S} s(i, N) \\
\geq & \sum_{i \in S} \sum_{j \in S: j<i} s(j, i, S)+\sum_{i \in S} s(i, S)=v(S)
\end{aligned}
$$

where the second inequality follows by Lemmas 5.2 and 5.3.

Also here, note that in fact the min-EGS core allocation is PMAS extendable by considering the min-EGS allocations for all subgames and the use of Lemmas 5.2 and 5.3.

Theorem 4.2 Min-RP sequencing games are convex.

Proof Let $\left(N, M, J, \sigma_{0}, p, \alpha\right)$ be a min-RP sequencing situation and let $(N, v)$ be the associated min-RP sequencing game. Note that the characteristic function of the game can be written as the sum of two characteristic functions: $v(S)=w(S)+u(S)$ with $w(S)=$ $\sum_{i \in S} \sum_{j \in S: i<j} s(i, j, S)$ and $u(S)=\sum_{i \in S} s(i, S)$. We show that both $(N, u)$ and $(N, w)$ are convex and therefore that $(N, v)$ is convex.

First, we prove that $(N, u)$ is convex. We have to show that

$$
u(S \cup\{j\})-u(S) \leq u(T \cup\{j\})-u(T)
$$

for every $j \in N$ and every $S \subset T \subset N \backslash\{j\}$.

Let $S \subset T$ and take $j \in N \backslash T$. Since,

$$
u(S \cup\{j\})-u(S)=\sum_{k \in S}(s(k, S \cup\{j\})-s(k, S))+s(j, S \cup\{j\})
$$

and

$$
u(T \cup\{j\})-u(T)=\sum_{k \in T}(s(k, T \cup\{j\})-s(k, T))+s(j, T \cup\{j\})
$$

it is sufficient to show that the following three inequalities are satisfied:

$$
\begin{gathered}
s(k, S \cup\{j\})-s(k, S) \leq s(k, T \cup\{j\})-s(k, T) \quad \text { for every } k \in S, \\
0 \leq s(k, T \cup\{j\})-s(k, T) \quad \text { for every } k \in T \backslash S, \\
s(j, S \cup\{j\}) \leq s(j, T \cup\{j\}) .
\end{gathered}
$$


Statement (1) is true by convexity of $s(*, \cdot)$ (Lemma 5.4) and statements (2) and (3) are true by monotonicity of $s(*, \cdot)$ (Lemma 5.2 ).

Secondly, we show that $(N, w)$ is convex. We have to show that

$$
w(S \cup\{j\})-w(S) \leq w(T \cup\{j\})-w(T)
$$

for every $j \in N$ and every $S \subset T \subset N \backslash\{j\}$.

Let $S \subset T$ and take $j \in N \backslash T$. Since

$$
\begin{aligned}
w(S \cup\{j\})-w(S)= & \sum_{k \in S} \sum_{i \in S: k<i}(s(k, i, S \cup\{j\})-s(k, i, S)) \\
& +\sum_{k \in S: k<j} s(k, j, S \cup\{j\})+\sum_{i \in S: j<i} s(j, i, S \cup\{j\})
\end{aligned}
$$

and

$$
\begin{aligned}
w(T \cup\{j\})-w(T)= & \sum_{k \in T} \sum_{i \in T: k<i}(s(k, i, T \cup\{j\})-s(k, i, T)) \\
& +\sum_{k \in T: k<j} s(k, j, T \cup\{j\})+\sum_{i \in T: j<i} s(j, i, T \cup\{j\})
\end{aligned}
$$

it is sufficient to show that

$$
\begin{aligned}
& s(k, i, S \cup\{j\})-s(k, i, S) \leq s(k, i, T \cup\{j\})-s(k, i, T) \\
& \quad \text { for every } k, i \in S, \text { with } k<i, \\
& 0 \leq s(k, i, T \cup\{j\})-s(k, i, T) \\
& \quad \text { for every } k \in T \backslash S, i \in T \text { or } k \in S, i \in T \backslash S, \text { with } k<i, \\
& s(k, j, S \cup\{j\}) \leq s(k, j, T \cup\{j\}) \quad \text { for every } k \in S, k<j, \\
& s(j, i, S \cup\{j\}) \leq s(j, i, T \cup\{j\}) \quad \text { for every } i \in S, j<i, \\
& 0 \leq s(k, j, T \cup\{j\}) \quad \text { for every } k \in T \backslash S, k<j, \\
& 0 \leq s(j, i, T \cup\{j\}) \quad \text { for every } i \in T \backslash S, j<i,
\end{aligned}
$$

Statement (4) is true by convexity of $s(*, *, \cdot)$ (Lemma 5.5). Statements (5), (6), and (7) are true by monotonicity of $s(*, *, \cdot)$ (Lemma 5.3). Finally, statements (8) and (9) follow since $s(*, *, \cdot)$ is nonnegative.

\section{Appendix}

Lemma 5.1 Let $S \subset T \subset N$. For every $i, j \in S$ with $i<j, r(i, j, S) \leq r(i, j, T)$.

Proof Let $\left(N, M, J, \sigma_{0}, p, \alpha\right)$ be a max-RP sequencing situation and take $i, j \in S$ with $i<j$. Let $i \in S_{r} \subset T_{\rho}$, with $S_{r}$ and $T_{\rho}$ components within the induced partition of $S$ and $T$, respectively. Moreover, let $U_{r}$ and $V_{\rho}$ be the correspondent components within the job collection associated to $S_{r}$ and $T_{\rho}$, respectively. Note that $U_{r} \subset V_{\rho}$. We distinguish two cases. 
Case 1: $j \notin S_{r}$. Here,

$$
r(i, j, S)=b_{i j}^{S_{r}}=\alpha_{i} \sum_{\substack{e \in J(j) \cap U_{r} \\ \sigma_{0}(e)<\sigma_{0}\left(l_{i}\right)}} p_{e} \leq \alpha_{i} \sum_{\substack{e \in J(j) \cap V_{\rho} \\ \sigma_{0}(e)<\sigma_{0}\left(l_{i}\right)}} p_{e}=b_{i j}^{T_{\rho}} \leq r(i, j, T),
$$

where the first inequality follows because $U_{r} \subset V_{\rho}$ and the second inequality follows by definition of $r(i, j, T)$.

Case 2: $j \in S_{r}$. Here, two subcases are considered.

Subcase 2.1: $g_{i j}^{S_{r}}=0$. Then

$$
r(i, j, S)=b_{i j}^{S_{r}}+g_{i j}^{S_{r}}=\alpha_{i} \sum_{\substack{e \in J(j) \cap U_{r} \\
\sigma_{0}(e)<\sigma_{0}\left(l_{i}\right)}} p_{e} \leq \alpha_{i} \sum_{\begin{array}{c}
e \in J(j) \cap V_{\rho} \\
\sigma_{0}(e)<\sigma_{0}\left(l_{i}\right)
\end{array}} p_{e}=b_{i j}^{T_{\rho}} \leq r(i, j, T)
$$

Subcase 2.2: $g_{i j}^{S_{r}}>0$. Then $l_{i}, l_{j} \in U_{r}$ and $g_{i j}^{S_{r}}=\alpha_{j} \sum_{d \in J(i) \cap U_{r}} p_{d}-\alpha_{i} \sum_{e \in J(j) \cap U_{r}} p_{e}$. Then,

$$
\begin{aligned}
r(i, j, T)= & b_{i j}^{T_{\rho}}+g_{i j}^{T_{\rho}} \geq \alpha_{i} \sum_{\substack{e \in J(j) \cap V_{\rho} \\
\sigma_{0}(e)<\sigma_{0}\left(l_{i}\right)}} p_{e}+\alpha_{j} \sum_{d \in J(i) \cap V_{\rho}} p_{d}-\alpha_{i} \sum_{e \in J(j) \cap V_{\rho}} p_{e} \\
= & \alpha_{i} \sum_{\substack{e \in J(j) \cap U_{r} \\
\sigma_{0}(e)<\sigma_{0}\left(l_{i}\right)}} p_{e}+\alpha_{i} \sum_{e \in J(j) \cap\left(V_{\rho} \backslash U_{r}\right)} p_{e}+\alpha_{j} \sum_{d \in J(i) \cap V_{\rho}} p_{d} \\
& -\alpha_{i} \sum_{e \in J(j) \cap U_{r}} p_{e}-\alpha_{i} \sum_{e \in J(j) \cap\left(V_{\rho} \backslash U_{r}\right)} p_{e} \\
= & b_{i j}^{S_{r}}+\alpha_{j} \sum_{d \in J(i) \cap V_{\rho}} p_{d}-\alpha_{i} \sum_{e \in J(j) \cap U_{r}} p_{e} \\
\geq & b_{i j}^{S_{r}}+\alpha_{j} \sum_{d \in J(i) \cap U_{r}} p_{d}-\alpha_{i} \sum_{e \in J(j) \cap U_{r}} p_{e} \\
= & b_{i j}^{S_{r}}+g_{i j}^{S_{r}}=r(i, j, S)
\end{aligned}
$$

where the first inequality follows by definition of $g_{i j}^{T_{\rho}}$ and the second equality follows because $l_{i}, l_{j} \in U_{r}, \sigma_{0}\left(l_{i}\right)<\sigma_{0}\left(l_{j}\right)$ and then $\left\{e \in J(j) \cap\left(V_{\rho} \backslash U_{r}\right) \mid \sigma_{0}(e)<\sigma_{0}\left(l_{i}\right)\right\}=$ $J(j) \cap\left(V_{\rho} \backslash U_{r}\right)$.

Lemma 5.2 Let $S \subset T \subset N$. For every $i \in S, s(i, S) \leq s(i, T)$.

Proof Let $\left(N, M, J, \sigma_{0}, p, \alpha\right)$ be a min-RP sequencing situation and let $i \in S$. Let $i \in S_{r} \subset$ $T_{\rho}$, with $S_{r}$ and $T_{\rho}$ components within the induced partition of $S$ and $T$, respectively. Moreover, let $U_{r}$ and $V_{\rho}$ be the components within the job collection associated to $S_{r}$ and $T_{\rho}$, respectively. Note that $U_{r} \subset V_{\rho}$. Hence,

$$
s(i, S)=\delta_{i i}^{S_{r}}=\alpha_{i}\left(p_{f_{i}}-\min _{d \in J(i) \cap U_{r}}\left\{p_{d}\right\}\right) \leq \alpha_{i}\left(p_{f_{i}}-\min _{d \in J(i) \cap V_{\rho}}\left\{p_{d}\right\}\right)=\delta_{i i}^{T_{\rho}}=s(i, T) .
$$

Lemma 5.3 Let $S \subset T \subset N$. For every $i, j \in S$ with $i<j, s(i, j, S) \leq s(i, j, T)$. 
Proof Let $\left(N, M, J, \sigma_{0}, p, \alpha\right)$ be a min-RP sequencing situation and let $i, j \in S$. Let $i \in S_{r} \subset T_{\rho}$, with $S_{r}$ and $T_{\rho}$ components within the induced partition of $S$ and $T$, respectively. Moreover, let $U_{r}$ and $V_{\rho}$ be the components within the job collection associated to $S_{r}$ and $T_{\rho}$, respectively. Note that $U_{r} \subset V_{\rho}$.

Case 1: $j \notin S_{r}$. Let $j \in S_{r^{*}} \subset T_{\rho^{*}}$ (with $U_{r^{*}} \subset V_{\rho^{*}}$ the associated components within the job collection). In this case $s(i, j, S)=\gamma_{i j}^{S_{r *}}$.

$$
s(i, j, S)=\gamma_{i j}^{S_{r^{*}}}=\alpha_{j} \sum_{\substack{d \in J(i) \cap\left(U_{\left.r^{*} \backslash\left\{f_{i}\right\}\right)} \\
\sigma_{0}(d)<\sigma_{0}\left(f_{j}\right)\right.}} p_{d} \leq \alpha_{j} \sum_{\begin{array}{c}
d \in J(i) \cap\left(V_{\rho^{*}} \backslash\left\{f_{i}\right\}\right) \\
\sigma_{0}(d)<\sigma_{0}\left(f_{j}\right)
\end{array}} p_{d}=\gamma_{i j}^{T_{\rho^{*}}} \leq s(i, j, T) .
$$

Case 2: $j \in S_{r} \subset T_{\rho}$. Here, $s(i, j, S)=\gamma_{i j}^{S_{r}}+\delta_{i j}^{S_{r}}+g_{i j}^{S_{r}}$ and $s(i, j, T)=\gamma_{i j}^{T_{\rho}}+\delta_{i j}^{T_{\rho}}+g_{i j}^{T_{\rho}}$. We distinguish two subcases.

Subcase 2.1: $g_{i j}^{S_{r}}=0$. Hence,

$$
\begin{aligned}
s(i, j, S) & =\gamma_{i j}^{S_{r}}+\delta_{i j}^{S_{r}}+g_{i j}^{S_{r}}=\gamma_{i j}^{S_{r}}+\delta_{i j}^{S_{r}} \\
& =\alpha_{j} \sum_{\substack{d \in J(i) \cap\left(U_{r} \backslash\left\{f_{i}\right\}\right) \\
\sigma_{0}(d)<\sigma_{0}\left(f_{j}\right)}} p_{d}+\alpha_{j}\left(p_{f_{i}}-\min _{d \in J(i) \cap U_{r}}\left\{p_{d}\right\}\right) \\
& \leq \alpha_{j} \sum_{\substack{d \in J(i) \cap\left(V_{\rho} \backslash\left\{f_{i}\right\}\right) \\
\sigma_{0}(d)<\sigma_{0}\left(f_{j}\right)}} p_{d}+\alpha_{j}\left(p_{f_{i}}-\min _{d \in J(i) \cap V_{\rho}}\left\{p_{d}\right\}\right) \\
& =\gamma_{i j}^{T_{\rho}}+\delta_{i j}^{T_{\rho}} \leq \gamma_{i j}^{T_{\rho}}+\delta_{i j}^{T_{\rho}}+g_{i j}^{T_{\rho}}=s(i, j, T) .
\end{aligned}
$$

Subcase 2.2: $g_{i j}^{S_{r}}>0$. In this case, $g_{i j}^{S_{r}}=\alpha_{j} \min _{e \in J(i) \cap U_{r}}\left\{p_{e}\right\}-\alpha_{i} \min _{d \in J(j) \cap U_{r}}\left\{p_{d}\right\}$ by definition. Therefore,

$$
\begin{aligned}
s(i, j, S)= & \gamma_{i j}^{S_{r}}+\delta_{i j}^{S_{r}}+g_{i j}^{S_{r}} \\
= & \alpha_{j} \sum_{\substack{d \in J(i) \cap\left(U_{r} \backslash\left\{f_{i}\right\}\right) \\
\sigma_{0}(d)<\sigma_{0}\left(f_{j}\right)}} p_{d}+\alpha_{j}\left(p_{f_{i}}-\min _{d \in J(i) \cap U_{r}}\left\{p_{d}\right\}\right) \\
& +\alpha_{j} \min _{\substack{d \in J(i) \cap U_{r} \\
\text { ind }}}\left\{p_{d}\right\}-\alpha_{i} \min _{e \in J(j) \cap U_{r}}\left\{p_{e}\right\} \\
= & \alpha_{j} \sum_{\substack{d \in J(i) \cap\left(U_{r} \backslash\left\{f_{i}\right\}\right) \\
\sigma_{0}(d)<\sigma_{0}\left(f_{j}\right)}} p_{d} \\
& +\alpha_{j} p_{f_{i}}-\alpha_{i} \min _{e \in J(j) \cap U_{r}}\left\{p_{e}\right\} \\
\leq & \alpha_{j} \sum_{\substack{d \in J(i) \cap\left(V_{j} \backslash\left\{f_{i}\right\}\right) \\
\sigma_{0}(d)<\sigma_{0}\left(f_{j}\right)}} p_{d}+\alpha_{j} p_{f_{i}}-\alpha_{i} \min _{e \in J(j) \cap V_{\rho}}\left\{p_{e}\right\} \\
\leq & \gamma_{i j}^{T_{\rho}}+\delta_{i j}^{T_{\rho}}+g_{i j}^{T_{\rho}}=s(i, j, T),
\end{aligned}
$$


where the first and second equalities follow by definition and the second inequality follows by definition of $g_{i j}^{T_{\rho}}$.

Lemma 5.4 Let $S \subset T \subset N$. For every $j \in N \backslash T$ and every $i \in S$,

$$
s(i, S \cup\{j\})-s(i, S) \leq s(i, T \cup\{j\})-s(i, T) .
$$

Proof Let $\left(N, M, J, \sigma_{0}, p, \alpha\right)$ be a min-RP sequencing situation. Let $i \in S$ and $j \in$ $N \backslash T$. Let $i \in S_{r} \subset T_{\rho}$, with $S_{r}$ and $T_{\rho}$ components within the induced partition of $S$ and $T$, respectively. Moreover, let $U_{r}$ and $V_{\rho}$ be the components within the job collection associated to $S_{r}$ and $T_{\rho}$, respectively. Let $i \in S_{t}^{(j)} \subset T_{\tau}^{(j)}$, with $S_{t}^{(j)}$ and $T_{\tau}^{(j)}$ components within the induced partition of $S \cup\{j\}$ and $T \cup\{j\}$, respectively. Besides, let $U_{t}^{(j)}$ and $V_{\tau}^{(j)}$ be the components within the job collection associated to $S_{t}^{(j)}$ and $T_{\tau}^{(j)}$, respectively. Note that $S_{r} \subset S_{t}^{(j)} \subset T_{\tau}^{(j)}, S_{r} \subset T_{\rho} \subset T_{\tau}^{(j)}, U_{r} \subset U_{t}^{(j)} \subset V_{\tau}^{(j)}$ and $U_{r} \subset V_{\rho} \subset V_{\tau}^{(j)}$.

Define fol $\left(U_{r}\right)$ to be the job that is processed in position $\max _{e \in U_{r}}\left\{\sigma_{0}(e)\right\}+1$. Note that fol $\left(U_{r}\right)$ may not exist. We distinguish two cases.

Case 1: fol $\left(U_{r}\right)$ does not belong to $j$. Here, we face the following situation:

\begin{tabular}{l|c|c|c|c|c}
\multicolumn{3}{c}{$f_{i}$} & \multicolumn{2}{c}{$\mathrm{fol}\left(U_{r}\right)$} \\
\hline & $\mathrm{i}$ & $\mathrm{m}$ & $\mathrm{i}$ & $\mathrm{m}$ & $\mathrm{m}$ \\
\hline
\end{tabular}

where $m \in S$ and the grid job either belongs to a player in $N \backslash\{j\}$ or represents the end of the queue. In this case, $J(i) \cap U_{r}=J(i) \cap U_{t}^{(j)}$ and the inequality comes down to $0 \leq$ $s(i, T \cup\{j\})-s(i, T)$, which is true by Lemma 5.2.

Case 2: fol $\left(U_{r}\right)$ belongs to $j$. In this case, we face the following situation:

\begin{tabular}{l|c|c|c|c|c|c|c}
\multicolumn{9}{c}{$f_{i}$} & \multicolumn{4}{c}{$\operatorname{fol}\left(U_{r}\right)$} \\
\hline & $\mathrm{i}$ & $\mathrm{m}$ & $\mathrm{i}$ & $\mathrm{m}$ & $\mathrm{m}$ & $\mathrm{j}$ & \\
\hline
\end{tabular}

where $m \in S$. As a result, $J(i) \cap U_{r}=J(i) \cap V_{\rho}$ and the inequality boils down to $s(i, S \cup$ $\{j\}) \leq s(i, T \cup\{j\})$, which follows by Lemma 5.2.

Lemma 5.5 Let $S \subset T \subset N$. For every $k \in N \backslash T$ and every $i, j \in S$ with $i<j$,

$$
s(i, j, S \cup\{k\})-s(i, j, S) \leq s(i, j, T \cup\{k\})-s(i, j, T) .
$$

Proof Let $\left(N, M, J, \sigma_{0}, p, \alpha\right)$ be a min-RP sequencing situation. Let $k \in N \backslash T$ and $i, j \in S$ with $i<j$. Let $i \in S_{r} \subset T_{\rho}$, with $S_{r}$ and $T_{\rho}$ components within the induced partition of $S$ and $T$, respectively. Moreover, let $U_{r}$ and $V_{\rho}$ be the components within the job collection associated to $S_{r}$ and $T_{\rho}$, respectively. Let $i \in S_{t}^{(k)} \subset T_{\tau}^{(k)}$, with $S_{t}^{(k)}$ and $T_{\tau}^{(k)}$ components within the induced partition of $S \cup\{k\}$ and $T \cup\{k\}$, respectively. Besides, let $U_{t}^{(k)}$ and $V_{\tau}^{(k)}$ be the components within the job collection associated to $S_{t}^{(k)}$ and $T_{\tau}^{(k)}$, respectively. Note that $S_{r} \subset S_{t}^{(k)} \subset T_{\tau}^{(k)}, S_{r} \subset T_{\rho} \subset T_{\tau}^{(k)}, U_{r} \subset U_{t}^{(k)} \subset V_{\tau}^{(k)}$ and $U_{r} \subset V_{\rho} \subset V_{\tau}^{(k)}$.

In order to show the statement, we study two cases.

Case 1: $j \in S_{r}$. Define fol $\left(U_{r}\right)$ to be the job that is processed in position $\max _{e \in U_{r}}\left\{\sigma_{0}(e)\right\}+$ 1. Note that fol $\left(U_{r}\right)$ may not exist. We distinguish two subcases. 
Subcase 1.1: fol $\left(U_{r}\right)$ does not belong to $k$. In this case we face the following situation:

\begin{tabular}{c|c|c|c|c|c|c|c}
\multicolumn{9}{c}{$f_{i}$} & \multicolumn{1}{c}{ fol $\left(U_{r}\right)$} \\
\hline & $\mathrm{i}$ & $\mathrm{m}$ & $\mathrm{i}$ & $\mathrm{j}$ & $\mathrm{m}$ & $\mathrm{j}$ & $\mathrm{m}$ \\
\hline
\end{tabular}

where $m \in S$ and the grid job either belongs to a player in $N \backslash\{k\}$ or represents the end of the queue. Here, $J(i) \cap U_{r}=J(i) \cap U_{t}^{(k)}$ and the inequality comes down to $0 \leq s(i, j, T \cup$ $\{k\})-s(i, j, T)$, which follows by Lemma 5.3.

Subcase 1.2: fol $\left(U_{r}\right)$ belongs to $k$. In this case we face the following situation:

\begin{tabular}{|c|c|c|c|c|c|c|c|}
\hline \multicolumn{2}{|c|}{$f_{i}$} & \multicolumn{2}{|r|}{$f$} & & & \multicolumn{2}{|c|}{ fol $\left(U_{r}\right)$} \\
\hline i & $\mathrm{m}$ & $\mathrm{i}$ & $\bar{j}$ & $\mathrm{~m}$ & $\mathrm{j}$ & $\mathrm{m}$ & $\mathrm{k}$ \\
\hline
\end{tabular}

where $m \in S$. As a result, $J(i) \cap U_{r}=J(i) \cap V_{\rho}$ and the inequality boils down to $s(i, j, S \cup$ $\{k\}) \leq s(i, j, T \cup\{k\})$, which is true by Lemma 5.3.

Case 2: $j \notin S_{r}, j \in S_{r^{*}} \subset T_{\rho^{*}}\left(j \in S_{t^{*}}^{(k)} \subset T_{\tau^{*}}^{(k)}\right)$.

$\operatorname{Define} \operatorname{pred}\left(U_{r^{*}}\right)$ to be the job that is processed in position $\min _{e \in U_{r^{*}}}\left\{\sigma_{0}(e)\right\}-1$. We distinguish two subcases.

Subcase 2.1: $\operatorname{pred}\left(U_{r^{*}}\right)$ does not belong to $k$. In this case we face the following situation:

\begin{tabular}{|c|c|c|c|c|}
\hline \# & J & $\mathrm{m}$ & J & $\mathrm{m}$ \\
\hline
\end{tabular}

where $m \in S$ and the grid job belongs to a player in $N \backslash\{k\}$. Here, $\left\{e \in J(i) \cap U_{r^{*}}: e<f_{j}\right\}=$ $\left\{e \in J(i) \cap U_{t^{*}}^{(k)}: e<f_{j}\right\}$ and the inequality comes down to $0 \leq s(i, j, T \cup\{k\})-s(i, j, T)$, which follows by Lemma 5.3.

Subcase 2.2: $\operatorname{pred}\left(U_{r^{*}}\right)$ belongs to $k$. In this case we face the following situation:

\begin{tabular}{r|r|r|r|r|r|r}
\multicolumn{2}{c}{$\operatorname{pred}\left(U_{r^{*}}\right)$} & \multicolumn{3}{l}{$f_{j}$} \\
\hline $\mathrm{k}$ & $\mathrm{m}$ & $\mathrm{j}$ & $\mathrm{m}$ & $\mathrm{j}$ & $\mathrm{m}$ & \\
\hline
\end{tabular}

where $m \in S$. As a result, $\left\{e \in J(i) \cap U_{r^{*}}: e<f_{j}\right\}=\left\{e \in J(i) \cap V_{\rho^{*}}: e<f_{j}\right\}$ and the inequality boils down to $s(i, j, S \cup\{k\}) \leq s(i, j, T \cup\{k\})$, which is true by Lemma 5.3.

\section{References}

Bondareva, O. N. (1963). Some applications of linear programming methods to the theory of cooperative games. Problemy Kibernitiki, 10, 119-139 (in Russian).

Calleja, P., Estévez-Fernández, A., Borm, P., \& Hamers, H. (2006). Job scheduling, cooperation and control. OR Letters, 34, 22-28.

Curiel, I., Pederzoli, G., \& Tijs, S. (1989). Sequencing games. European Journal of Operational Research, 40, 344-351. 
Curiel, I., Hamers, H., \& Klijn, F. (2002). Sequencing games: a survey. In P. Borm \& H. Peters (Eds.), Chapters in game theory: honor of Stef Tijs (pp. 27-50). Boston: Kluwer Academic.

Shapley, L. S. (1967). On balanced sets and cores. Naval Research Logistics Quarterly, 14, 453-460.

Shapley, L. S. (1971). Cores of convex games. International Journal of Game Theory, 1, 11-26.

Smith, W. (1956). Various optimizers for single-stage production. Naval Research Logistics Quarterly, 3, 59-66.

Sprumont, Y. (1990). Population monotonic allocation schemes for cooperative games with transferable utility. Games and Economic Behavior, 2, 378-394. 